\title{
STI com adaptação de interface e estratégias pedagógicas
}

\author{
Rudimar Luís Scaranto Dazzi ${ }^{1}$, Fernando Medes de Azevedo ${ }^{2}$, Ana Paula Soares \\ Fernandes $^{3}$ \\ ${ }^{1}$ Grupo de Inteligência Aplicada, Centro de Ciências Tecnológicas da Terra e do Mar, \\ Universidade do Vale do Itajaí. Rua Uruguai, 458, Itajaí-SC, Brasil \\ ${ }^{2}$ Instituto de Engenharia Biomédica, Departamento de Engenharia Elétrica, \\ Universidade Federal de Santa Catarina. Campus Trindade, CEP: 80040-900, \\ Florianópolis-SC, Brasil \\ ${ }^{3}$ Departamento de Estomatologia, Universidade Federal de Santa Catarina. Campus \\ Trindade, CEP: 80040-900, Florianópolis-SC, Brasil \\ rudimar@univali.br, azevedo@ieb.ufsc.br, anapaulasoaresfernandes@yahoo.com.br
}

\section{Resumo}

O foco deste artigo é apresentar uma metodologia para a construção de Sistemas Tutores Inteligentes (STI) com adaptação de interface e de estratégia pedagógica de forma independente e complementar. Esta metodologia permite a construção de STI com múltiplas estratégias pedagógicas (EP) sendo utilizadas simultaneamente, adaptando-se ao perfil do estudante. De forma complementar a interface se adapta ao estudante, seguindo um perfil determinado com a teoria das inteligências múltiplas (IM). São apresentados ao estudante os conteúdos com mídias computacionais na proporção considerada adequada pela teoria das IM e na EP determinada ao estudante. Os testes realizados apontam a metodologia como promissora.

Palavras-Chave: Sistemas Tutores Inteligentes, Sistemas educacionais adaptativos, Metodologia e arquitetura.

\section{Abstract}

\section{ITS with interface and pedagogic strategy adaptation}

This paper present a methodology to develop Intelligent Tutors Systems (ITS). It adapts the interface and pedagogic strategy in an independent and complementary way. This methodology is based on multiple pedagogic strategies (PE) through adapting from the student's profile. Also, the interface adapts to the student according to the theory of the multiple intelligences (MI). The contents is presented to the student by computational media as theory of MI and the best PE to the student. The accomplished tests point the methodology as promising.

Keywords: Intelligent tutoring systems; Adaptative educational systems; Methodology and architecture.

\section{Introdução}

Os avanços tecnológicos aliados aos paradigmas educacionais, produziram uma transformação no processo tradicional de ensino e aprendizagem, determinando exigências pedagógicas cada vez maiores. No ambiente tecnológico da educação, para que se consiga fazer com que o aluno absorva o conteúdo, não basta apresentar-lhe os conteúdos corretamente, é necessário prender sua atenção e apresentar as informações em um contexto adequado. As aplicações computacionais para a educação deixaram de 
2

ser simples reprodutoras de informações para tentar mudar os métodos de ensino/aprendizagem e redefinir os objetivos educacionais e resultados do aprendizado (Cumming \& Mcdougall, 2000).

Sistemas de apoio à aprendizagem, como os Sistemas Tutores Inteligentes (STI) ou os Sistemas Hipermídia Adaptativos (SHA), têm sido desenvolvidos com o intuito de disponibilizar as informações aos alunos e simultaneamente apresentar mecanismos capazes de se adequar às características do aluno, a fim de mostrar-se efetivo no processo de ensino e aprendizagem. No entanto, a maioria destes sistemas incorpora, de uma forma ou de outra, um só modelo pedagógico como estratégia de ensino (Oliveira e Fernandes, 2003 e 2004; Barbosa 2004; Curilem e De Azevedo, 2002). Os modelos pedagógicos utilizados nestes sistemas podem ser tanto aqueles descritos na literatura quanto os definidos de forma empírica (DE Oliveira et al. 2005).

Neste aspecto, este trabalho apresenta uma alternativa para a construção de STI, por meio de uma metodologia (apresentada sinteticamente neste artigo) que os torna adaptativos. Esta adaptação se dá em dois momentos, na interface e na estratégia pedagógica, uma vez que propõe a utilização de múltiplas mídias e múltiplas estratégias pedagógicas em um só sistema de ensino. Na busca por melhores resultados, foi utilizada uma abordagem híbrida na construção destes sistemas, agregando à proposta, as interfaces adaptativas (Curilem e De Azevedo, 2002; Barbosa 2004) e os agentes pedagógicos (De Azevedo, Dazzi, Fernandes e Fernandes, 2005) de forma independente.

O desafio desta é manter, de forma coerente e harmônica, as múltiplas mídias a serem tratadas pela interface adaptativa com as múltiplas estratégias pedagógicas (EP), que também vão adaptar-se ao perfil dos alunos. Os agentes, neste contexto, são responsáveis por controlar cada uma das diferentes EP e definir dentre elas qual a mais apropriada para o aluno.

\section{Detalhamento do modelo de arquitetura de desenvolvimento proposta}

Para alcançar o objetivo de obter um sistema de ensino que seja mais eficiente, adaptando-se aos alunos e suas características, propôs-se uma abordagem um tanto arrojada, separando o tratamento de interface, do tratamento das estratégias pedagógicas (EP) utilizadas no sistema. Observando os trabalhos da área, verificou-se que todos pretendem de alguma forma adaptar o sistema ao aluno, mas sempre utilizando EP vinculadas à interface.

Este modelo inspirou-se na estrutura dos STI (Vicari e Giraffa, 2003; Self, 1999), mas com independência entre a apresentação visual dos conteúdos (interface), e a estratégia de ensino, responsável pela organização de como os conteúdos são abordados e disponibilizados aos alunos.

Com o intuito de encontrar soluções para esta divisão e, principalmente, para conseguir disponibilizar em uma só ferramenta de ensino diversas abordagens pedagógicas, buscou-se as soluções nos conceitos de agentes (Garcia e Sichman, 2003; Franceschi et al, 2002; Russel e Norvig, 2004). Os agentes foram organizados em comunidade para obter os resultados esperados, no que se refere às múltiplas EP. Neste caso, cada agente é implementado para responder a uma EP, sendo que é possível inserir tantos agentes quantos forem necessários, para atender a todas as EP desejadas, conforme pode ser visualizado no modelo apresentado na Figura 1. 
3

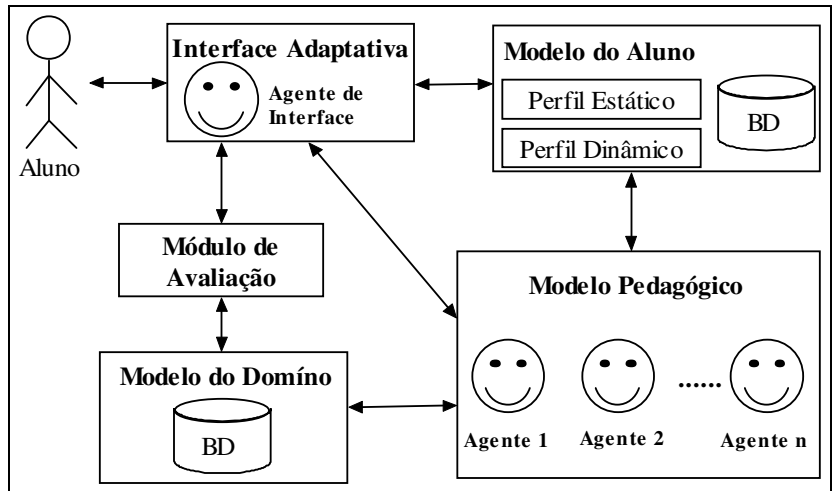

Figura 1: Representação Gráfica do Modelo Proposto.

O modelo apresentado na Figura 1, possui módulos similares aos dos STI tradicionais, separando o domínio, as estratégias, o perfil do aluno e a interface. Porém aqui, existem algumas diferenças básicas, na forma de como está se propondo o Modelo Pedagógico e o tratamento das interfaces, ambos adaptativos ao aluno. A seguir discutiremos cada um dos componentes do modelo proposto.

\section{Modelo Pedagógico}

No modelo pedagógico, os múltiplos agentes responsáveis por cada uma das EP abordadas precisam de um mecanismo cognitivo que permita a tomada de decisão necessária para atender aos preceitos da estratégia deste agente. Este módulo também necessita decidir, dentre as estratégias disponibilizadas no sistema, qual delas deve ser empregada para cada aluno. A arquitetura deste módulo pode ser analisada na Figura 2.

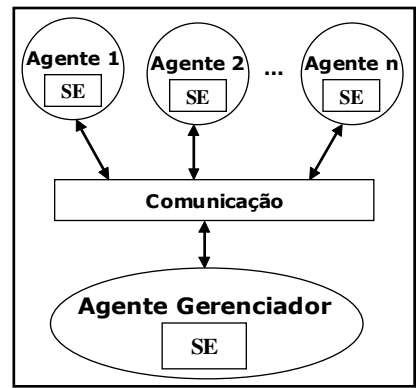

Figura 2: Modelo Pedagógico Ampliado e Detalhado

Os agentes do modelo pedagógico podem ser caracterizados segundo Russel e Norvig (2004) como sendo "agentes reativos baseados em modelo", pois mantêm um estado interno, que é utilizado na análise das situações futuras. E possuem algumas características de classificação (Garcia e Sichman, 2003) tais como: comunicabilidade, pela interação entre os agentes de estratégia com o agente gerenciador, na busca pela definição da estratégia a ser utilizada e da responsabilidade de tutoriamento do aluno; quanto ao ambiente de atuação, estes agentes atuam na internet, mas no ambiente específico de cada aluno; e podem ser considerados reativos, visto que detectam as ações do usuário e agem de acordo com elas.

Esses agentes possuem seu mecanismo cognitivo baseado em Sistema Especialista (SE), fundamentado em regras de produção. Estas regras são definidas pedagogicamente por um especialista, para atender a estratégia do agente. Um agente especial nesse modelo foi denominado de Agente Gerenciador, responsável por definir 
4

qual das EP é a mais apropriada para cada aluno, definindo assim qual dos Agentes de EP deve conduzir os estudos do aluno.

\section{Modelo do Aluno}

O modelo do Aluno é responsável por detectar e manter o perfil do aluno com suas características. Para determinar o perfil do aluno quanto à adaptação ao tipo de mídia que os conteúdos serão apresentados, é utilizada a Teoria das Inteligências Múltiplas (IM). Esta teoria foi escolhida por acreditar-se que se as informações forem apresentadas de forma a atender as características do perfil do aluno, este se sentirá mais motivado a continuar seus estudos e assimilará os conteúdos com maior eficiência.

Para a determinação das características do aluno, este precisa preencher um teste de múltipla escolha, o qual é apresentado na primeira vez que o aluno acessa o sistema (Dazzi, 2007). Este teste permite que sejam definidos os valores das IM dos alunos para que possa ser realizada a adaptação inicial do sistema (processo de adaptatividade do sistema). Para implementar a adaptação na interface, utiliza-se quatro inteligências, dentre todas as propostas por Gardner (2001), na identificação das características do aluno. Estas inteligências são: Lingüístico-verbal (relacionada às palavras e à linguagem escrita e falada); Lógico-matemática (relacionada ao raciocínio científico, intuitivo e dedutivo); Visual-espacial (relacionada a habilidade de criar imagens mentais) e Cinestésico-corporal (relaciona-se com o movimento físico).

As respostas obtidas do questionário são submetidas a uma rede neural artificial (RNA) multi layer perceptron (MLP) que tem o objetivo de transformar as 30 respostas (relativas às $4 \mathrm{IM}$ ) em 4 notas correspondentes, respectivamente, a nota da IM Lingüístico-verbal, Lógico-matemática, Visual-espacial e Cinestésico-corporal. Com as notas do aluno geradas, outra RNA MLP que as utiliza como entrada, gera as quantidades de mídias para cada IM.

Após a utilização das duas RNA MLP, o perfil inicial do aluno (para interface) está completo, pois foram geradas as notas de cada uma das 4 inteligências múltiplas baseadas nas respostas do questionário de avaliação inicial e nas 4 quantidades de conteúdos a serem apresentados em cada uma das mídias. Para que o sistema possa iniciar a apresentação dos conteúdos, ainda é necessário que se faça uma distribuição da ordem de apresentação dos conteúdos em cada uma das 4 mídias. Isso é feito de forma aleatória (por sorteio), pois a teoria das inteligências múltiplas não indica nenhum tipo de distribuição mais ou menos adequada a cada caso, o importante é disponibilizar os conteúdos em diferentes mídias nas proporções indicadas (Dazzi, 2007).

Além das características do aluno para a adaptação a interface, é preciso obter o perfil necessário para a determinação da estratégia pedagógica a ser seguida pelo aluno em seu estudo. Para fazer a escolha inicial da estratégia pedagógica para o aluno, também são feitas algumas perguntas relativas ao grau de conhecimento que este aluno possui do assunto a ser estudado (Dazzi, 2007).

Como a estratégia pedagógica também é dinâmica e pode ser alterada de acordo com os resultados obtidos pelo aluno em seus estudos no sistema, não há uma preocupação maior na identificação perfeita deste perfil no primeiro momento. Vale destacar aqui que a identificação da estratégia pedagógica é feita analisando o cadastro inicial do aluno, mas vai ser alterada sempre que os agentes julgarem oportuno, de acordo com os resultados das análises das ações do aluno pelo SE.

\section{Interface Adaptativa}


5

Para adequar as 4 IM ao tipo de mídia computacional a ser utilizada na interface, foram feitos alguns estudos (Armstrong, 2000; Antunes, 2001; Campbell, Campbell e Dickinson, 1998; Gardner, 1993; Gardner, 2001; Curilem e De Azevedo, 2002; Barbosa, 2004) que remetem às mídias de texto para a IM Lingüístico-verbal; aos esquemas gráficos, como fluxogramas, organogramas e mapas conceituais para a IM Lógico-matemática; às figuras, fotos e desenhos para a IM Visual-espacial; e às animações, hiperlinks e vídeos para a IM Cinestésico-corporal.

O conteúdo apresentado ao aluno não é apenas em uma das mídias, mas sim distribuído entre as mídias, de acordo com a recomendação pedagógica e baseado no perfil do aluno, tendo em vista que cada indivíduo possui desenvolvimento em cada uma das inteligências, mas com um grau diferente. Com isso, a IM com maior desenvolvimento no aluno será a que definirá a maior quantidade de material na mídia correspondente. Todas as mídias são utilizadas em todos os alunos, mas em quantidades diferentes, adequando assim ao seu perfil de interface (relativo às IM).

Para manter o perfil do aluno sempre atualizado, baseado não só no resultado do questionário inicial, mas também nas escolhas feitas pelo aluno durante a navegação no sistema, utilizam-se as redes neurais artificiais do tipo IAC (Interactive Activation and Competition) (Sigaki, De Azevedo e Barreto, 1997; Stevenage e Lewis, 1999; Rumelhart e Mcclelland, 1986; De Azevedo, 1993) que, por possuírem característica de bidirecionalidade, permitem que se faça a readequação do perfil de interface.

Para atender a esta necessidade, o sistema identifica todas as escolhas do aluno e repassa a informação a uma rede IAC que faz a avaliação da situação atual corrigindo ou não a quantidade de mídias de cada conteúdo apresentado ao aluno. Como a rede IAC é bidirecional, esse processo pode ser tratado adequadamente, pois tanto podem ser ativados na rede as quantidades de mídias para obter as notas das IM do aluno como podem ser ativadas as notas das IM para obter a quantidade de mídias na mesma rede IAC. Este é um dos principais motivos para a utilização deste tipo de rede para resolver esse problema.

Com esse modelo foi possível gerar a adaptação da interface ao perfil do usuário. Como o processo é interativo e se reajusta à medida que o usuário altera a forma de apresentação proposta pelo sistema, redefinindo dessa forma o perfil do aluno. Com isso, se houver algum desvio na identificação inicial do perfil do aluno, isso será automaticamente readequando nos primeiros passos do aluno no sistema, quando esse solicitar mudanças nas mídias apresentadas.

\section{Modelo do Domínio}

Neste modelo são armazenados os conteúdos que serão abordados no curso de forma a permitir a apresentação destes aos alunos nas diversas estratégias pedagógicas implementadas pelo sistema. No STI desenvolvido para validar a metodologia, foram utilizadas 2 EP e o modelo de banco de dados (BD) utilizado está atendendo às necessidades, pois prevê a divisão dos conteúdos em pequenas porções que podem ser administradas nas EP para atender os requisitos de cada uma delas. O STI desenvolvido para validar a metodologia, teve como área de aplicação a odontologia e utilizou duas EP, uma baseada em casos clínicos e outra denominada tradicional, que segue o padrão tradicional de aulas ministradas por professores.

Os conteúdos relativos à inteligência lógico-matemática devem ser feitos por meio de esquemas, testes lógicos e afins. Uma das formas mais tradicionais de se representar isso na área tecnológica é pelo uso de fluxogramas e árvores de decisão, 
6

neste caso como o conhecimento é de odontologia optou-se por utilizar os mapas conceituais (Pozo, 2002; Lima, 2004; Sue et al, 2004).

Os conteúdos relativos à IM cinestésico-corporal devem ser feitos utilizando animações ou interações do usuário com o conteúdo. Neste caso, pela própria prática da área de odontologia que costuma utilizar filmes, foi utilizado, principalmente, este recurso nos conteúdos. Foram feitos filmes de procedimentos realizados nas clínicas e utilizaram-se estes filmes para esta mídia.

Os conteúdos relativos à IM visual-espacial foram feitos de duas formas: com imagens criadas para representar o fato em questão e com fotos extraídas de trabalhos publicados de materiais de odontologia.

Os conteúdos em texto relativos à IM linguistico-verbal foram feitos sem problemas, pois não exige nenhum recurso especifico, e para sua confecção basta o especialista repassar os conteúdos a serem utilizados.

\section{Módulo de Avaliação}

O módulo de avaliação é responsável por montar, controlar, avaliar e dar retorno ao modelo pedagógico sobre os resultados das avaliações de cada aluno. Baseado nos resultados das avaliações, é que serão revistos os perfis dos alunos para readequar ou não a EP utilizada, adaptando ao estilo do aluno.

O resultado das avaliações não é utilizado por este módulo, mas é utilizado pelos agentes para analisar o prosseguimento dos estudos do aluno. Estes resultados são analisados pelas regras dos agentes e estes definem se o aluno segue para outra unidade ou retorna os estudos para revisão. No caso da necessidade do aluno rever os conteúdos, ele será submetido novamente à avaliação após a revisão para novamente avaliar seu conhecimento. Neste caso, é necessário que este módulo apresente outras questões de avaliação e/ou as mesmas questões já apresentadas, mas neste caso a seqüência de respostas é apresentada em uma ordem diferente, para evitar a memorização de respostas por posição e não por conteúdo.

\section{Discussões e resultados}

Nesse trabalho, pretendeu-se também confirmar a tendência de melhoria no aprendizado de alunos que utilizam STI semelhante aos estudos realizados por Soh (2006) e Feng, Heffernan e Koedinger (2006). Com a metodologia proposta com a dupla adaptação ao perfil do aluno, o desempenho e, consequentemente, o ganho de aprendizagem torna-se equivalente ao apresentado por Soh (2006).

Alguns exemplos de interface com conteúdo, utilizadas no STI desenvolvido com esta metodologia são apresentados na seqüência. Na Figura 3, são apresentados dois exemplos de telas com conteúdos na EP tradicional e nas mídias esquema e animação, neste caso, filme de um procedimento odontológico. 


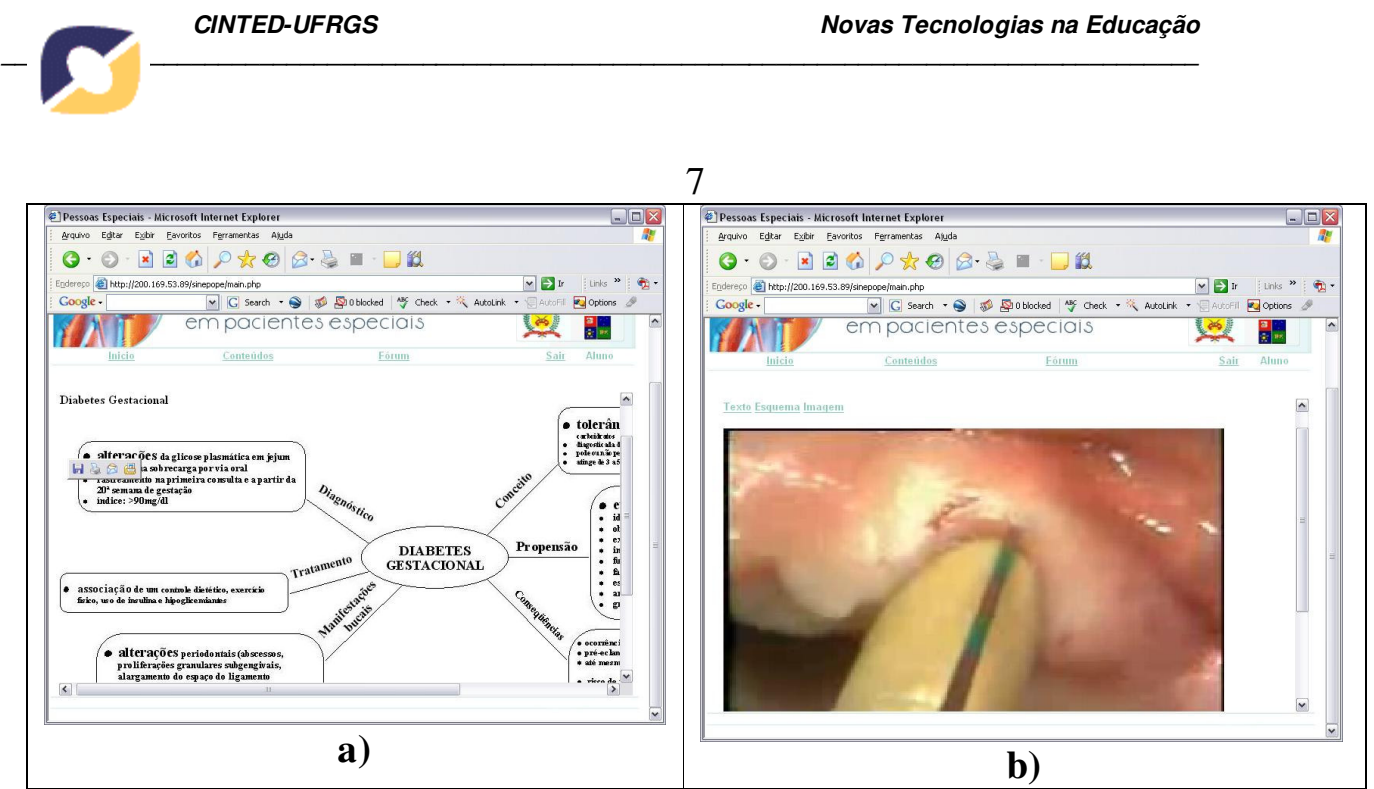

Figura 3: Exemplo de conteúdo da EP tradicional nas mídias esquema (a) e animação (b).

Além dos conteúdos da EP tradicional, também existem os conteúdos da EP casos clínicos. Estes conteúdos seguem o mesmo principio de utilização de mídias, sendo apresentado na Figura 4 dois exemplo representando respectivamente as mídias texto e figura (imagem).

Com o objetivo de avaliar a adequação do STI ao usuário e analisar os aspectos pedagógicos deste e, conseqüentemente, da metodologia proposta, ele foi apresentado a alunos do curso de Odontologia, para utilização e avaliação. Foi realizada uma análise para identificar se a utilização do STI apresenta resultados similares a um professor ministrando o mesmo conteúdo. Para tal, os alunos participantes desta pesquisa foram divididos em dois grupos, um com 14 alunos que tiveram uma aula expositiva com um professor e outro com 15 alunos que utilizaram o STI (do total de 29 da amostra). Esta divisão foi feita de forma proporcional por período cursado pelos alunos, considerando aqui que estes alunos possuem desempenho similar entre seus pares no curso.

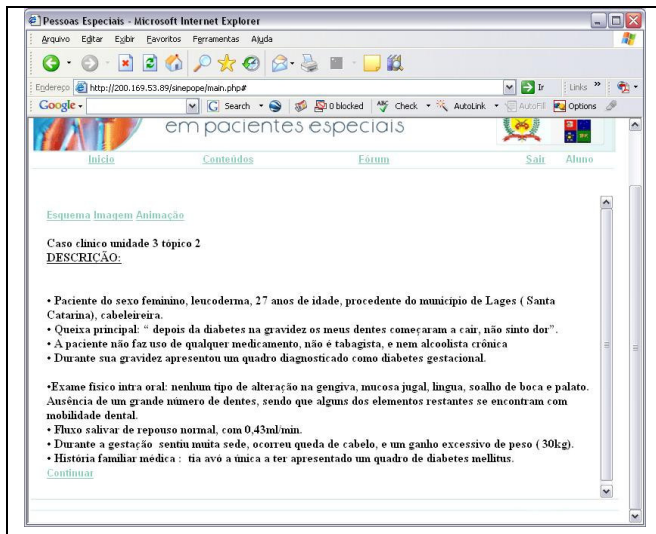

a)

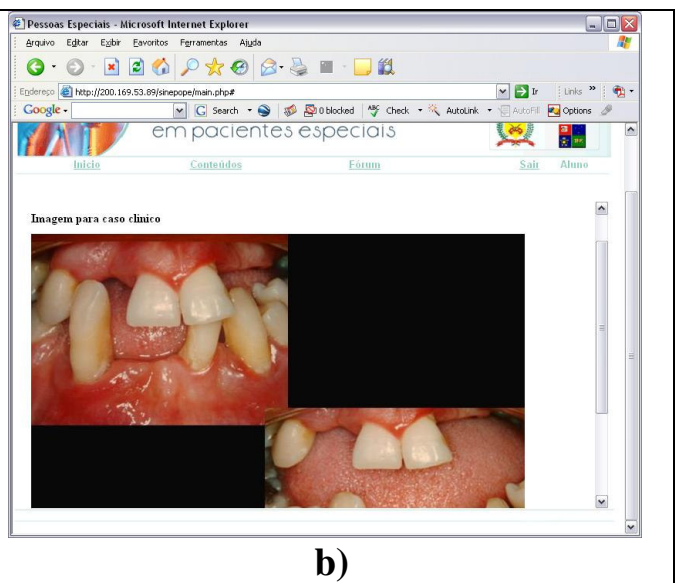

b)

Figura 4: Exemplo de conteúdo da EP casos clínicos nas mídias texto (a) e imagem (b).

Para avaliar o resultado geral dos alunos em ambas as turmas, foram analisadas as médias da avaliação por aluno, o que permitiu verificar o desempenho dos alunos. Os alunos de ambos os grupos responderam as mesmas perguntas. Como o STI proporciona V. 6 № 1 , Julho, 2008 
a revisão dos conteúdos em parte ou totalmente para os alunos que não conseguiram bons resultados, utilizou-se aqui, apenas as respostas da primeira avaliação feita pelo STI.

O gráfico da Figura 5 apresenta a média de cada um dos 14 alunos que participaram da aula expositiva ministrada por um professor, que resultou numa média geral da turma de 5,56 pontos (considerou-se a faixa de notas de 0 a 10). Deve-se considerar aqui que esta avaliação foi efetuada imediatamente após a conclusão da aula, o que não permitiu que os alunos fizessem qualquer tipo de reflexão ou revisão dos conteúdos. Esse fato deve ter contribuído para o resultado final da média da turma (abaixo de 7,0), mas foi realizado desta maneira visando manter o teste o mais uniforme possível com os dois grupos avaliados.

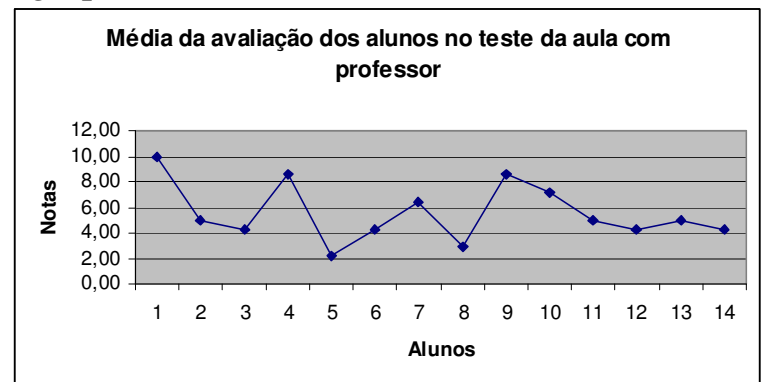

Figura 5: Gráfico da média da avaliação dos alunos com o professor.

No caso do grupo que utilizou o STI, a média geral da turma foi de 6,24 pontos, ressaltando que fora utilizando o resultado da primeira avaliação, para se equivaler ao teste feito com o primeiro grupo. Os resultados da médias por aluno podem ser observados no gráfico da Figura 6.

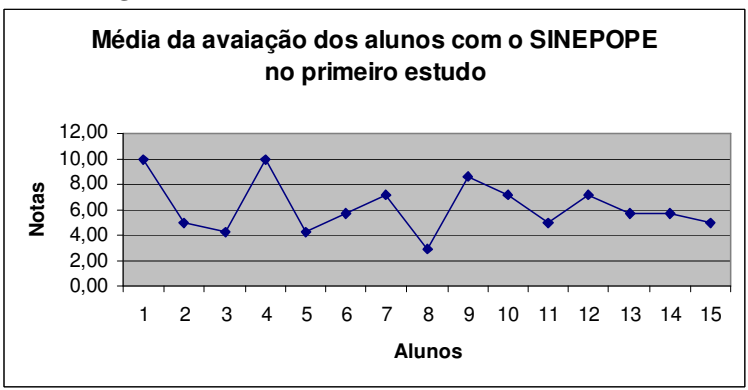

Figura 6: Gráfico da média da avaliação dos alunos com o SINEPOPE.

Apesar de o resultado ter sido ligeiramente melhor do que o primeiro grupo não é possível afirmar que o sistema teve um desempenho melhor que o professor. No entanto, pode-se considerar que o sistema também conseguiu fazer com que os alunos se apropriassem dos conteúdos. Todos os testes e avaliações realizados podem ser vistos em detalhes em Dazzi (2007).

\section{Conclusões}

A proposta de tratar de forma independente as interfaces das estratégias pedagógicas do sistema visa aprimorar o nível de adaptabilidade geral do sistema ao aluno. Outro ponto relevante dessa metodologia é que permite que se tenham tantas EP quantas se deseja, podendo ser incluídas novas EP no sistema sem a necessidade de refazer o que já foi feito. Sendo necessário apenas fazer alguma modificação nas regras 
9

do SE do gerenciador do modelo pedagógico e acrescentar novos materiais característicos dessa EP.

Os resultados dos testes e avaliações realizados indicam que o sistema desenvolvido utilizando a metodologia proposta proporcionou aos alunos a apropriação dos conteúdos de forma similar ao ocorrido na aula com um professor. Em virtude de não ser possível e viável no momento realizar um estudo ao longo de um semestre e com mais conteúdos no sistema, torna-se difícil afirmar com plena convicção que os alunos que utilizam o sistema se saiam sempre melhor que alunos que assistam a aulas tradicionais. Porém, com os resultados verificados, pode-se dizer que há indícios fortes de que a utilização do sistema pode suprir as necessidades de ensino dos alunos.

Esses indícios podem ser confirmados com os resultados de aprendizagem apresentados, onde o resultado das avaliações feitas com os alunos que utilizaram o sistema foi ligeiramente melhor do que as mesmas avaliações realizadas com os alunos que não utilizaram o sistema, mas submetidos a uma aula com professor.

Acredita-se com base em todos os estudos e resultados obtidos, que o futuro desta metodologia seja promissor, proporcionando mais uma alternativa eficiente de gerar soluções de ensino por computador para atender às necessidades de mercado.

\section{Referências}

Antunes, Celso. 2001. Como Identificar em Você e em Seus Alunos as Inteligências Múltiplas. Fascículo 4. Editora Vozes, Petrópolis, Brasil.

Armstrong, Thomas. 2000. Multiple Intelligences in the Classroom. Association for Supervision \& Curriculum Deve, 2nd. ed., USA, pp. 95 - 120.

Barbosa, A. T. R. 2004. Mecanismo de Adaptação Baseado em Redes Neurais Artificiais para Sistemas Hipermídia Adaptativa. Tese de doutorado. Universidade Federal de Santa Catarina. Departamento de Pós-graduação em Engenharia Elétrica. Florianópolis.

Campbell, L.; Campbell, B.; Dickinson, D. 1998. Teaching and Learning Through Multiple Intelligences, Allyn \& Bacon. 2nd. ed., pp. 320 - 340.

Cumming, G. \& McDougall, A. 2000. Mainstreaming AIED into education?

International Journal of Artificial Intelligence in Education, 11, 197-207.

Curilem, G. M. J.; De Azevedo, F. M. 2002. Ergonomia Didática na Interface de

Sistemas Tutores Inteligentes. Anais do Congresso Ibero-americano de Informática Educativa - IE2002, Vigo-Espanha.

Dazzi, R. L. S. 2007. Metodologia para Adaptação de Interface e Estratégia Pedagógica em Sistemas Tutores Inteligentes. Tese de doutorad $o$. Universidade Federal de Santa Catarina. Departamento de Pós-graduação em Engenharia Elétrica. Florianópolis.

De Azevedo, F. M.; Dazzi, R. L. S.; Fernandes, A. P. S.; Fernandes, A. M. da R. 2005. Metodologia para Sistemas Tutores Inteligentes: Aplicação para Pacientes Especiais em Odontologia. In: IADIS 2005, 2005, Lisboa. Actas da Conferência IADIS IBERO-AMERICANA. Lisboa: Editores Associados: Luís Rodrigues e Patrícia Barbosa. v. 1, p. 223-230.

De Azevedo, F. 1993. Contribution to the Study of Neural Networks in Dynamical Expert Systems. Institute d'Informatique - FUNDP. Namour, Belgium. 
10

DE Oliveira, E. da S. G.; Santos, L.; Costa, M. de A.; Villardi, R. M. 2005. A Avaliação na Educação a Distância: Reflexões e Estratégias para o Ensino Universitário. Anais do XII Congresso Internacional de Educação a Distância. Florianópolis-SC.

Feng, M.; Heffernan, N. T.; Koedinger, K. R. 2006. E-learning \& scientific applications: Addressing the testing challenge with a web-based e-assessment system that tutors as it assesses. Proceedings of the 15th international conference on World Wide Web WWW '06. ACM Press.

Franceschi, A. S. M.; et al. 2002. Desenvolvendo Agentes moveis para Gerência de Redes Utilizando Técnicas de Inteligência Artificial. Anais do Congresso Brasileiro de Computação - CBComp 2002. Itajaí, Brasil, pp. 26-30.

Garcia, A. C. B.; Sichman, J. S. 2003. Agentes de Sistemas Multiagentes. In: BARONE, Dante (org.). Sociedades Artificiais. Bookman, Porto Alegre, Brasil. p.270-306

Gardner, H. 2001. Estrutura da Mente. Editora Artmed, Porto Alegre, Brasil.

Lima, G. Â. B. 2004. Mapa Conceitual como ferramenta para organização do conhecimento em sistema de hipertextos e seus aspectos cognitivos. Perspectivas em Ciência da Informação v. 9 no. 2 (July/December 2004) p. 134-145

Oliveira, J. M. P.; Fernandes, C. T. 2003. A Framework for Adaptive Educational Hypermedia Systems. Workshop on Applications, Products and Services of Webbased Support Systems in conjunction with the IEEE/WIC, Anais... International Conference on Web Intelligence, Halifax, Canada, pp. 55-62.

Oliveira, J. M. P.; Fernandes, C. T., 2004, Instructional Model as a Driving Force for Adaptation in Adaptive Educational Hypermedia Systems, Proceedings of EDMedia 2004, World Conference on Educational Multimedia, Hypermedia \& Telecomunications, AACE.Lugano, Switzerland.

Pozo, J. I. 2002. Aprendizes e Mestres: a nova cultura da aprendizagem. Editora Artmed, Porto Alegre, Brasil.

Rumelhart, D. E.; Mcclelland J. L. 1986. Explorations in Parallel Distributed Processing. volume 1. Foundations, The MIT Press.

Russel, S.; Norvig, P. 2004. Inteligência Artificial: uma abordagem moderna. Editor Campus, São Paulo, Brasil.

Self, J. A. 1999. The defining characteristics of intelligent tutoring systems research: ITSs care, precisely. International Journal of Artificial Intelligence in Education, v10, p. 350-364.

Sigaki, N.A; De Azevedo, F. M.; Barreto, J. M. 1997. Enhancing Connectionist Expert Systems Using IAC Models Through Real Cases - Proceeding of The ICANNGA 97- 3TH International Conference on Artificial Neural Networks and Genetic Algorithms, Norwick, UK.

Stevenage, S. V.; Lewis, H. G. 1999. IACAPA: modelling recognition and learning of people with an interactive activation and competition model. In: Artificial Neural Networks. Anais ICANN 99 - Ninth International Conference on Edinburgh, UK. (Vol 2, pp 779-784).

Soh, Leen-Kiat. 2006. Incorporating an intelligent tutoring system into CS1. ACM SIGCSE Bulletin, Proceedings of the 37th SIGCSE technical symposium on Computer science education SIGCSE '06. ACM Press, 2006. v.38.

Sue, P.C.; Weng, J.F.; SU, J.M.; Tseng, S.S. 2004. A new approach for constructing the concept map. Proceedings. IEEE International Conference on Advanced Learning Technologies. 2004, p. 76-80 
11

Vicari, R. M.; Giraffa, L. M. M. 2003. Fundamentos dos Sistemas Tutores Inteligentes. In: Barone, D. (org). Sociedades artificiais: a nova fronteira da inteligência das máquinas. Bookman, Porto Alegre, Brasil. 